\title{
Educación rural y la adquisición de competencias laborales: una innovación mediante el uso de recursos digitales
}

\section{Rural Education and the Acquisition of Occupational Skills: An Innovative Experience through the Use of Digital Resources}

\author{
Efrén Salgado Vivas ${ }^{1}$ \\ efrensalgado1@hotmail.com \\ Marcela Georgina Gómez-Zermeño \\ marcela.gomez@itesm.mx \\ María Manuela Pintor Chávez \\ maria.pintor@tecvirtual.mx \\ Instituto Tecnológico y de Estudios Superiores de Monterrey, México
}

\section{Resumen:}

El presente artículo muestra un estudio realizado con el objetivo de conocer las percepciones de los participantes sobre la implementación de recursos tecnológicos en las instituciones educativas para el desarrollo de competencias laborales en el sector agrícola. Se realizó la implementación de dos recursos tecnológicos: Redes Sociales y Biblioteca Digital. La investigación se llevó a cabo aplicando el método mixto en un diseño convergente paralelo, por medio de tres instrumentos: encuesta de caracterización, entrevista a grupo focal, y encuesta de satisfacción de herramientas, en tres Instituciones ubicadas en el Departamento de Cundinamarca, Colombia, donde participaron 63 estudiantes de entre los 14 y 17 años de edad de los

\begin{abstract}
:
This article reports on a study conducted in rural areas in order to understand the perceptions of the participants on the implementation of two technological resources in educational institutions for skills development in the agricultural sector: Social Networks and Digital Library. The research was carried out by applying the mixed method in a parallel convergent design, using three instruments: characterization survey, focus group interview, and satisfaction survey. The experiment was undertaken in three institutions located in the Department of Cundinamarca, Colombia and 63 students between 14 and 17 years of age from 10th grade participated. The results allowed to establish three criteria which influence innovation
\end{abstract}

1 Dirección para correspondencia (correspondence address):

Efrén Salgado Vivas. Instituto Tecnológico y de Estudios Superiores de Monterrey. Eugenio Garza Sada 2501, Col. Tecnológico. Monterrey, Nuevo León (México) 
Educación rural y la adquisición de competencias laborales: una innovación mediante el uso de recursos digitales

Efrén Salgado Vivas, Marcela Georgina Gómez-Zermeño y María Manuela Pintor Chávez

grupos del grado $10^{\circ}$. Los resultados permitieron establecer tres criterios que influyen en procesos de innovación mediante el uso de herramientas tecnológicas. El primer criterio se relaciona con las Competencias Laborales, las cuales generan oportunidades, arraigo y sentido de pertenencia de los estudiantes frente a sus regiones y sitios de ubicación y también generan opciones de mejor calidad de vida. El segundo criterio se estableció con respecto a los contenidos para la interacción de los estudiantes deben manejar temas relevantes. El tercer criterio se refiere al mejoramiento de la estructura tecnológica, ya que las falencias en conectividad limitan el manejo de los recursos tecnológicos. Se concluye que es necesario adaptar los procesos educativos a las condiciones y contexto de los estudiantes.

\section{Palabras clave:}

Competencia profesional; enseñanza técnica; enseñanza agrícola; estudiante de secundaria; joven. processes when technological tools are used. The first criterion relates to labor skills which generate opportunities, roots and sense of belonging of students regarding their regions and location as well as better quality of life. The second criterion was established with respect to how elicit student interaction-the topics used should be relevant to students. The third criterion relates to the improvement of the technological means, since connectivity shortcomings limit the use of technological resources. The study concludes that it is necessary to adapt the educational process to the conditions and context of students.

\section{Key words:}

Occupational qualifications; Technical education; Agricultural education; Secondary school students; teenager.

\section{Résumé:}

Cet article présente une étude menée dans les zones rurales afin de comprendre les perceptions des participants sur la mise en œuvre des ressources technologiques dans les établissements d'enseignement pour le développement des compétences dans le secteur agricole. Deux ressources technologiques ont été mises en œuvre : Réseaux sociaux et Bibliothèque numérique. La recherche a été effectuée en appliquant la méthode mixte dans une conception convergente parallèle, en utilisant trois instruments: enquête de caractérisation, interview d'un groupe de discussion, et des outils d'enquête de satisfaction, dans trois établissements situés dans le département de Cundinamarca, la Colombie, où ont participé 63 étudiants âgés de 14 à 17 ans du groupe de d 10ème année. Les résultats ont permis $d^{\prime}$ 'établir trois critères qui influent sur les processus d'innovation en utilisant des outils technologiques. Le premier critère concerne les compétences de la main-d'œuvre, qui génèrent des opportunités, des racines et le sentiment d'appartenance des élèves par rapport à leurs régions et lieux de localisation et procurent une meilleure qualité de vie. Le deuxième critère a été établi en ce qui concerne le contenu de l'interaction des étudiants devrait traiter des questions pertinentes. Le troisième critère concerne aussi bien I'amélioration de la structure technologique, que les lacunes de connectivité limitée à la gestion des ressources technologiques. Nous concluons qu'il est nécessaire d'adapter le processus éducatif aux conditions et au contexte des étudiants.

\section{Mots clés:}

Qualification professionnelle; enseignement technique; enseignement agricole; élève du secondaire; jeune.

Fecha de recepción: 05-06-2015

Fecha de aceptación: 09-11-2016 
Efrén Salgado Vivas, Marcela Georgina Gómez-Zermeño y María Manuela Pintor Chávez

\section{Introducción}

En la actualidad, los sistemas de producción agrícola en Colombia se someten a diversas complicaciones, por ejemplo al respecto de posibilidades de trabajo e ingreso, ya que en el campo existe el problema de la baja calidad del empleo y bajas entradas económicas (Leibovich, Nigrinis y Ramos, 2005). Algunas de esas problemáticas están asociadas a obsoletas formas de fabricación, elaboración, transformación y comercialización de productos agropecuarios, desde el punto de vista tradicional y cultural (Cuesta, 2008), que concibe un bajo desarrollo en el cubrimiento de las necesidades básicas del sector. Por lo tanto, es necesario fomentar nuevas técnicas de producción, modernizar e implementar tecnologías y conocimientos específicos que lleven al progreso y el bienestar de sus actores, sin olvidar la importancia del adecuado manejo de los recursos naturales (Ospina, 2008).

Existen dificultades para mejorar la calidad de la educación ya que actualmente persisten los modelos de escuela tradicional basados en la instrucción presencial centrada en el profesor. De igual manera, es necesaria la muestra de interés de los maestros en participar en procesos que conduzca a nuevos e innovadores modelos de aprendizaje.

Por los motivos mencionados, es necesario implementar una estrategia que permita mejorar los procesos educativos mediante la implementación de estrategias innovadoras. La implementación de las Tecnologías de la Información y la Comunicación (TIC) en educación genera grandes beneficios; no obstante su simple implementación no implica el mejoramiento en la calidad de la educación. Las TIC cobran sentido cuando existe una conexión entre los miembros de la comunidad educativa: maestros, directivos, padres de familia, estudiantes, sector productivo y sociedad en general. Dichos actores deben comprometerse en un proceso de enseñanza - aprendizaje contextualizado y participar en una preparación tecnológica que fomente el espíritu innovador en los estudiantes (Alemán, Gómez-Zermeño, Parada y Sainz, 2011).

Actualmente, el maestro demanda una práctica renovada que fortalezca el proceso, como indica McKinsey (2007, p. 40) "la calidad de los resultados para cualquier sistema educativo es esencialmente el resultado de la calidad de la instrucción brindada por sus docentes". El profesor debe asumir el rol de volver funcional las herramientas para que la calidad de la educación Ilegue al aula. Asimismo, el rol del estudiante presto 
Educación rural y la adquisición de competencias laborales: una innovación mediante el uso de recursos digitales

Efrén Salgado Vivas, Marcela Georgina Gómez-Zermeño y María Manuela Pintor Chávez

al cambio, dejando el papel tradicional de receptor pasivo y convertirse en un receptor activo, con motivación propia para el estudio (Cabero, 2006). Las TIC en la educación necesitan versatilidad y adecuación al cambio que estimule su aprovechamiento, deben incorporarse como elemento movilizador a entornos propicios para el aprendizaje (Vargas, Gómez-Zermeño y Gómez-Zermeño, 2013).

\section{Educación Rural y la Adquisición de Competencias Laborales}

El proyecto de Competencias Laborales, está enmarcado en la política de articulación de la educación media con el mundo productivo, responde a la necesidad del país de tener personas versátiles, creativas y con capacidad de innovación, abiertas a nuevos aprendizajes y dispuestas a trabajar en equipo en un entorno específico (Portal Colombia Aprende, 2007). Las competencias laborales son todos los conocimientos, habilidades y actitudes, necesarios para que los jóvenes se desempeñen eficientemente en procesos productivos (MEN, 2007).

Las competencias laborales se dividen en generales (CLG) para formación desde la educación básica hasta la media, y las especificas (CLE) para desarrollar en la educación media técnica, buscando la formación para el trabajo y la articulación con la educación superior. Las CLG son el conjunto de conocimientos, habilidades, actitudes y valores que los estudiantes deben desarrollar para su desempeño apropiado en cualquier entorno productivo, sin importar el sector económico, el nivel del cargo, la complejidad de la tarea o el grado de responsabilidad. Con las CLG, un joven actúa asertivamente, sabe trabajar en equipo, tiene sentido ético, maneja de forma acertada los recursos, puede solucionar problemas y aprende de las experiencias de los otros. Las CLE orientan la formación de un individuo para realizar funciones productivas propias de una ocupación o conjunto de ocupaciones organizadas principalmente por la necesidad del sector de ubicación, pero también por afinidad temática, necesidades personales y de la sociedad. En el caso de Colombia, esta educación es ofrecida por las IE de educación media técnica.

Un aspecto importante es la formación de ciudadanos con capacidad de decisión democrática, con valores sociales, personas críticas y creativas capaces de enfrentar y resolver problemas cotidianos; que entregue como resultado, entre otros el contribuir al mejoramiento de la calidad 
de vida presente y futura, y, de esta manera la mejor composición de la sociedad. Es necesario instruir a los estudiantes de acuerdo a la realidad de su contexto, por lo tanto es de suma importancia enfocarlo al manejo de los elementos que se encuentran en el entorno (Schmelkes, 1994).

Conforme lo anterior, las IE Rurales deben buscar una formación técnica o formación por competencias laborales enfocadas a las necesidades del contexto, creando estudiantes que vean el campo como una alternativa viable, deseable, lúdica, ambientalmente amigable, pero sobretodo como un contexto lleno de desarrollo tanto de conocimientos como de tecnología. Un proceso formativo donde se promueva el aprender por implementación de nuevas tecnologías, además de estimular la capacidad de enfrentarse y afrontar los desafíos productivos, generando retos en la calidad de la producción, transformación y comercialización de bienes agropecuarios (Santiesteban, 2008).

\section{Implementación de Innovación Educativa}

Según Carbonell (citado en Rimari, 1996, p.3), la innovación educativa es "un conjunto de ideas, procesos y estrategias, más o menos sistematizados, mediante los cuales se trata de introducir y provocar cambios en las prácticas educativas vigentes". El propósito de la innovación educativa es la transformación de los procesos de enseñanza y aprendizaje.

La primera solución, es la implementación de la tecnología en las nuevas formas de aprendizaje, que implica cambios en el que aprender y en lo que hacen docentes y estudiantes dentro y fuera de las aulas y las instituciones (Correa y De Pablos, 2009). De la misma forma, señalan la importancia que tiene la introducción de las TIC en el contexto educativo para el impulso de la pedagogía, estimulando a la búsqueda de nuevas formas de aprendizaje.

La educación en el entorno rural permite ver el campo como una opción de vida, brindando acceso y posibilidades a pequeños productores y empresarios del campo para organizar actividades productivas y empleos de calidad, con capital social e infraestructura de importancia estratégica en la estabilidad democrática del país (Leibovich, Nigrinis y Ramos, 2005). Maestros comprometidos con la comunidad, con actitud crítica y capacidad para analizar el contexto rural desde el punto de vista cultural, social, económico, educativo e institucional, que den 
Educación rural y la adquisición de competencias laborales: una innovación mediante el uso de recursos digitales

Efrén Salgado Vivas, Marcela Georgina Gómez-Zermeño y María Manuela Pintor Chávez

respuesta a los problemas identificados en el sector (Ministerio de Educación Nacional, 2002).

En esta investigación se plantea la pregunta: ¿Cómo las Redes Sociales a manera de herramienta tecnológica y de innovación, pueden fortalecer las prácticas de enseñanza en la educación media técnica y el aprendizaje de las competencias laborales específicas en las áreas de producción en las Instituciones Educativas (IE) rurales del Departamento de Cundinamarca?

Así, el objetivo del estudio será identificar la manera en que las redes sociales permiten el fortalecimiento de la enseñanza de competencias laborales específicas en áreas de producción, transformación y comercialización de bienes de origen agropecuario en las IE rurales en el nivel de Educación Media Técnica en el Departamento de Cundinamarca. Se propone la posibilidad de utilizar las redes sociales como una propuesta estratégica de implementación tecnológica en la forma de enseñar en el nivel medio técnico, con un enfoque en las necesidades del contexto, para formar estudiantes que ven el campo como una alternativa viable, deseable, lúdica y ambientalmente amigable.

Los estudiantes que aprenden interactuando en las redes sociales son capaces de enfrentarse a la búsqueda de soluciones a problemas reales y sirven para descubrir capacidades y destrezas propias; generando cambios y desafíos en la producción, transformación y comercialización de bienes agropecuarios (Santiesteban, 2008). De esta manera, en este estudio se considera importante fomentar cambios en el que aprender sobre lo que hacen docentes y estudiantes dentro y fuera de las aulas (Correa y De Pablos, 2009), permite ayudar a solucionar algunas de las problemáticas de los sistemas de producción rural en Colombia (Cuesta, 2008).

\section{Método}

El método mixto o también Ilamado tercer movimiento metodológico, fue utilizado en la presente investigación para establecer un proceso con datos tanto cuantitativos como cualitativos, mezclados de forma coherente. El tipo de diseño seleccionado es el convergente paralelo el cual lleva a cabo en forma simultánea componentes cualitativos y cuantitativos, de la misma manera se hace la entrega de los resultados finales (Valenzuela y Flores, 2011). 
Se llevó a cabo la selección de instrumentos; donde se hace distinción entre datos cualitativos y cuantitativos para poder realizar una mezcla en la información; ya sea cualificar los datos cuantificados o cuantificar los datos cualificados facilitando la comparación y el análisis (GómezZermeño, 2009). Teniendo en cuenta el protocolo, los instrumentos para la recolección de datos fueron tres:

- Cuestionario de caracterización para conocer el perfil familiar de los participantes, nivel educativo, acceso a medios tecnológicos e Internet.

- Grupo Focal: se realizó la observación a participantes llevadas a cabo en varios momentos del tiempo, para establecer tendencias de la población o el desarrollo evolutivo. La observación es una técnica de colección de datos cuyo propósito es describir eventos, situaciones y comportamientos (Valenzuela y Flores, 2011).

- Encuestas para conocer el nivel de satisfacción con respecto al manejo de los recursos tecnológicos que se utilizaron en la investigación, los cuales se presentan a continuación.

Las herramientas tecnológicas (HT) a utilizar en la investigación son las siguientes:

1) Redes sociales. (En adelante RS) Son comunidades virtuales, donde se interactúa buscando afinidad. Funcionan como una plataforma de comunicaciones, permitiendo centralizar recursos como fotos, videos, foros, etc., de tal manera que todas las personas pueden acceder de forma fácil. El medio propicio es el Internet, ya que permite la interconexión (Castro, 2014).

2) Biblioteca Digital. (En adelante BD). Fue otra HT implementada para la investigación. Una BD se ha planteado como el nuevo ideal de la organización de una colección de datos digitales más o menos organizados, que sirve a una comunidad especifica con las mismas intenciones. Se trata de recursos informáticos documentales con formato digital, de forma que se integra la técnica informática y las comunicaciones. La información ofrecida suele encontrarse en formatos; pdf, doc, jpg, bmp, mp3, entre otros (Tramullas, 2011). 
Educación rural y la adquisición de competencias laborales: una innovación mediante el uso de recursos digitales

Efrén Salgado Vivas, Marcela Georgina Gómez-Zermeño y María Manuela Pintor Chávez

\section{Población y muestra}

Para indagar sobre el problema de investigación se tomaron tres Instituciones Educativas de nivel medio técnico, pertenecientes a carreras del campo:

- Institución Educativa Departamental Técnico Agroindustrial Bateas. Ubicada en la Inspección de Bateas. Municipio de Tibacuy - Región del Sumapaz.

- Institución Educativa Departamental Cartagena. Ubicada en la Inspección El Ocaso. Municipio de Zipacón - Región de Sabana Occidente.

- Institución educativa Departamental Claraval Chuscales. Ubicada en la Inspección Claraval Chuscales. Municipio de Junín - Región de Guavio.

Como muestra se seleccionaron los grupos de los grados $10^{\circ}$ de las tres instituciones (primer año de la educación media), resultando en 63 estudiantes entre los 14 y 17 años de edad y de los cuales se pueden obtener datos importantes en cuanto a proyección de vida y sus intereses frente a la educación como instrumento de desarrollo personal.

Se llevó a cabo de forma simultánea a su aplicación antes, durante y después de la implementación; los datos colectados de forma cuantitativa se proceden a organizar por categorías en gráficos; así mismo, los datos cualitativos se comparan en cuanto a situaciones y puntos de vista de cada participante. El análisis se realiza cruzando la información vista en cada estudiante de cada IE para la validación de la información haciendo conclusiones con datos tanto cualitativos como cuantitativos. La metodología utiliza un diseño de triangulación, que de acuerdo con Creswell (1994) citado por Gómez-Zermeño (2009), la cual es una estrategia en la cual el objeto de estudio es abordado en diferentes momentos temporales para analizar sus avances y perspectivas.

\section{Resultados}

Como se señaló en la sección de Método, en este estudio se aplicaron tres instrumentos. A continuación se presentan los resultados obtenidos en cada aplicación. 


\section{Cuestionario de caracterización}

El instrumento cuestionario de caracterización permitió conocer el perfil poblacional, desde las áreas personales, familiares y algunas características para dar planeación al estudio. En cuanto a la situación familiar de los estudiantes, $84.6 \%$ afirmaron pertenecer a una familia funcional, mientras que $7.77 \%$ mencionó vivir con familiares. $69.2 \%$ afirma habitar en vivienda propia, $23 \%$ en arriendo y $7.7 \%$ comparte su hogar. Con respecto al tema de investigación, es interesante resaltar que $61.5 \%$ de los participantes habita en vivienda tipo finca.

Se evidencia hegemonía de vivienda propia y residencia en familias funcionales (padres, hijos y hermanos). Al ser estudiantes de IE rurales, las viviendas son fincas con pequeñas extensiones. Posteriormente, se indagó sobre el nivel educativo en el núcleo familiar de los participantes. La figura 1 muestra que tanto padres como madres poseen en mayor medida solamente el nivel de primaria.

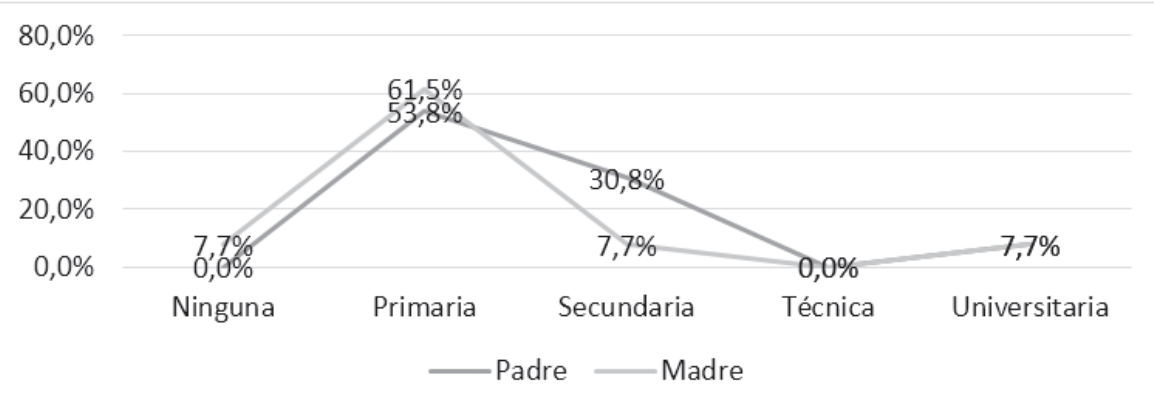

\section{Figura 1. Nivel de Educación del Núcleo Familiar.}

Los padres con mayor educación, le dan más importancia a que sus hijos manejen herramientas tecnológicas y tengan facilidad de acceso a internet. Posteriormente, se buscó conocer el acceso a la tecnología desde los hogares. Se logró conocer con que herramientas cuenta la población estudiada, así como expectativas familiares frente al uso de las mismas y sus posibilidades reales de funcionalidad.

En cuanto al acceso a medios tecnológicos propios, los estudiantes en un $45 \%$ mencionaron contar con estas herramientas mientras que $54 \%$ no. Con respecto a la misma pregunta a las familias, en un $85 \%$ respondieron que no tienen acceso a medios tecnológicos en sus 
hogares, mientras que un 15\% sí. Respecto este último porcentaje, se evidenció prioridad de los padres por compra de HT, especialmente computadoras y tablet para sus hijos, con la idea de mejorar la calidad en el proceso de aprendizaje. Los estudiantes que comentan poseer herramientas, es la única que se tiene en la vivienda, convirtiéndose en HT familiar.

Con respecto al acceso a internet, en su mayoría lo realizan a diario $(84.6 \%)$, rompiendo lo que se pudiera pensar al estar en una zona rural. $76.9 \%$ utiliza la conexión del hogar y $15.3 \%$ acude al café internet. Al indagar a fondo sobre lo que sucede respecto al acceso a internet, se encontró que práctica común descargan software para la navegación gratuita en algunas páginas, especialmente redes sociales, pero no navegación en la Web. Asimismo, se les cuestionó sobre el propósito de uso del Internet, y en su mayoría afirmaron realizar actividades de aprendizaje o para comunicarse, especialmente redes sociales (Figura 2).

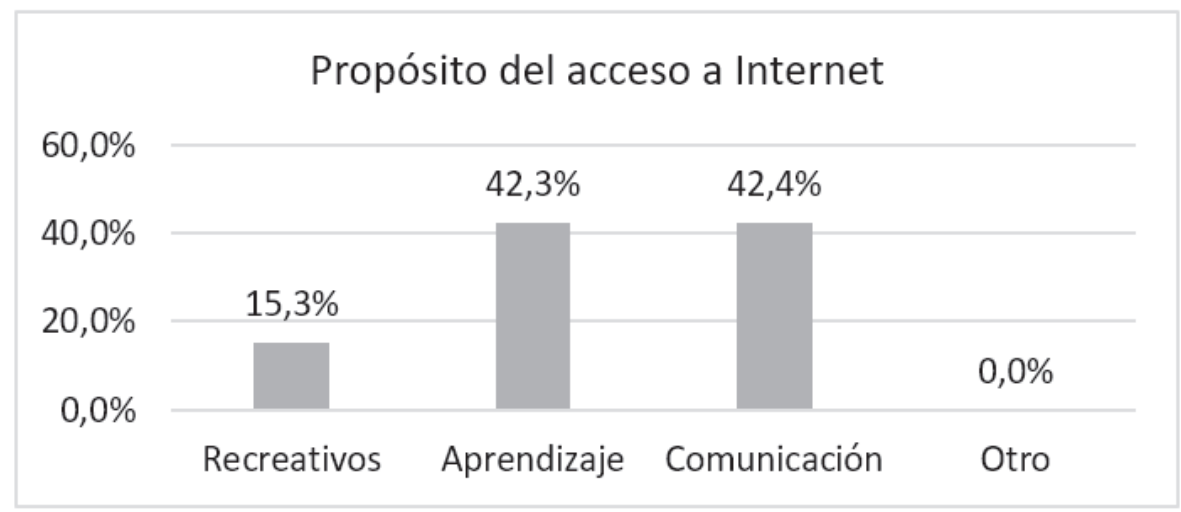

Figura 2. Acceso a Internet.

Un hallazgo que se obtuvo mediante la encuesta, es que para la muestra no es importante conocer y manejar muchas herramientas que ofrece el computador; la prioridad es saber navegar en internet desde distintos dispositivos. Asimismo, para la muestra con posibilidad de manejo permanente de herramientas, no es tan importante el uso en la educación. 


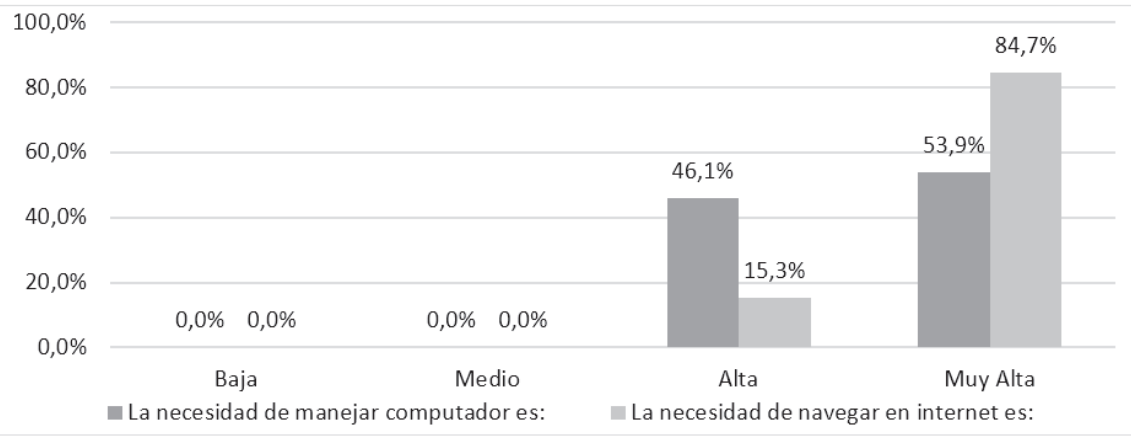

Figura 3. Percepción de Importancia.

Al preguntar a estudiantes si sabían cuántos dispositivos existen en sus instituciones, mencionaron entre 10 y 30, pero menos de diez están a disposición de estudiantes y docentes. En relación a este aspecto, se preguntó a docentes el por qué de esta percepción de la población estudiantil y uno explicó que en Colombia existe la asociación Ilamada "Computadoras para Educar", la cual promueve las TIC como factor de desarrollo equitativo y sostenible (Computadoras para Educar, 2013). Dicha entidad, hizo entrega a la institución de 50 computadoras, sin embargo, a pesar de contar en promedio con más de 50 computadoras, no se cuenta con un mantenimiento preventivo, ocasionando fallas en su sistema operativo y así el número de equipos disponibles se reduce. De igual forma, mencionaron que no cuentan con mobiliario adecuado para el manejo lo cual genera vulnerabilidad por caídas y malos manejos. Por último, la carencia más importante es la falta de conectividad y por este motivo no se están usando de forma relevante en las distintas áreas del conocimiento.

En una profundización para esta pregunta, se comprobó el interés de los docentes por aprender a manejar medios tecnológicos y hacer uso en sus clases. Entre los principales hallazgos de este instrumentos fueron:

- La posibilidad de tener conectividad permanente y de calidad en las IE rurales en el Departamento de Cundinamarca, por diversos motivos es baja, lo que afecta la implementación de cualquier HT que necesite uso de internet.

- Las personas adultas tienen claro que se está en la era del conocimiento globalizado y la importancia de interactuar con otras culturas, pero tienen miedo a los contenidos no favorables para los estudiantes. 
- Es muy baja la capacitación a estudiantes sobre el manejo del internet. La población en investigación piensa que el dominio de Facebook, Twitter, Google es suficiente para ser un experto, lo que genera deficiencias en su utilización.

- Se evidenció, que si bien, existen políticas gubernamentales para la implementación de equipos tecnológicos en las IE a nivel nacional, existen falencias de seguimiento y control.

- También, al preguntar a los docentes, por qué la percepción de los estudiantes de las fallas en el manejo de los medios tecnológicos por parte de ellos, argumentan que existe falta de capacitación, pero es alto el interés por adquirir conocimientos en el manejo de HT. Una apreciación que tienen los estudiantes es que las TIC no son importantes como recurso educativo, ya que el docente solo las utiliza para disminuir el esfuerzo personal y no las hace significativas en aplicación en diversas áreas.

- Se necesita la creación e implementación de un Manual Ético para el manejo de TIC en Educación, que genere responsabilidad por parte de los actores.

\section{Entrevista a Grupo Focal}

El segundo instrumento para recolección de datos correspondió a una entrevista a grupo focal en torno a sus expectativas del uso de las herramientas tecnológicas. Por grupos de estudiantes, se debatió y se sintetizaron las respuestas. La implementación de las HT se ejecutó con el fin de hacer observación y analizar expectativas por los participantes. Las diez preguntas realizadas al grupo focal se presentan a continuación, con relación a la calidad de la herramienta tecnológica, calidad didáctica, metodológica y técnica.

1. ¿Qué tan importante en cuanto a actualidad, innovación y creatividad fue la implementación de una HT para mejorar la calidad de la educación media técnica?

Como estudiantes, se conoce el cambio acelerado que genera el manejo de TIC en educación, que a su vez genera retos a los actores para ofrecer un enorme potencial de transformación en la educación. Sin embargo, para dichos cambios, los docentes deben diseñar, implementar y evaluar experiencias de aprendizaje por medio de TIC. 
Efrén Salgado Vivas, Marcela Georgina Gómez-Zermeño y María Manuela Pintor Chávez

Así mismo, los estudiantes están dispuestos a:

- Llevar a cabo aprendizaje con creatividad e innovación.

- Trabajar en equipo, siempre en aras de la colaboración interpersonal.

- Investigación efectiva para búsqueda de información.

- Pensar de forma crítica, solucionando problemas y tomando decisiones.

- Uso de TIC de forma adecuada y ética.

2.En cuanto a la eficacia y eficiencia ¿qué opinan sobre cómo las HT ayudaron a mejorar la calidad de la educación media técnica?

La educación es lo más importante que tiene una sociedad, posee múltiples desafíos que solo la unión de los actores lo puede solucionar. Es importante implementar estas y otras HT, que busquen la innovación en el proceso de aprendizaje de forma eficaz y eficiente.

3. ¿La HT es versátil y fácil de adaptar a las necesidades personales y sociales?

Como dice el argot popular, los estudiantes en la era moderna viene con el chip insertado; aunque esta frase es solo un concepto distraído del manejo de la tecnología en las nuevas generaciones, el estudiante tiene la percepción de facilidad para aprender el manejo de cualquier HT; eso sí, el docente debe ser una persona creativa e innovadora que cause impacto y despierte curiosidad y necesidad de uso por parte de los estudiantes.

En cuanto a las HT, la BD, tiene su falencia en la limitación al manejo independiente de cada IE, mientras la RS tiene crecimiento permanente debido a la participación; el inconveniente, es la conectividad.

4. ¿La HT fue atractiva y la propondrían nuevamente en otras áreas?

Es lógico pensar que una HT exitosa en un área específica, se aplique en otras áreas; es el caso de la BD, donde la población hace énfasis en establecerla no solamente para el área técnica, sino para todas las áreas institucionales. En cuanto a la RS, se desenvuelve mejor si solo comparte información especializada, lo lógico puede ser que se tenga dentro de la Red Social Educativa un enfoque a cada área del conocimiento.

5. ¿La HT facilitó la interacción entre todos los participantes?

Como se resalta, las redes sociales facilitaron los encuentros sincrónicos y asincrónicos, generando expectativas para la exposición de documentos, fotografías, videos y demás recursos compartidos como experiencias exitosas. Contrario a esto, con el uso de la biblioteca digital solo 
Educación rural y la adquisición de competencias laborales: una innovación mediante el uso de recursos digitales

Efrén Salgado Vivas, Marcela Georgina Gómez-Zermeño y María Manuela Pintor Chávez

se pudo Ilevar a cabo en cada IE de forma independiente. Se apreció que las RS, no solo se usan como medio para compartir información personal, sino para interactuar y la construcción del conocimiento.

6. ¿La didáctica utilizada para la implementación, estuvo acorde con la HT?

Aunque es normal, al inicio se generó resistencia al cambio, es allí donde los docentes deben tener estrategias didácticas para despertar interés. Es precisamente lo que permitió en las IE la implementación de las HT; aunque después de un tiempo los estudiantes estaban más atraídos por la RS, a pesar de que el estudio de cada HT se hacía independiente, los estudiantes en cada IE compartían avances y se encontró que era fuerte el impacto de la RS para su implementación, frente a la BD que después de un tiempo se convirtió en monotemática para algunos estudiantes.

7. ¿La metodología utilizada para la implementación de la HT fue suficiente y eficiente para el desarrollo de la misma?

El conocimiento avanzado de las HT por parte del investigador, facilito su implementación, ya que en ningún momento los estudiantes se sintieron navegando sin rumbo, contrario, se despertó intereses e ideas del manejo de las mismas. Aunque si bien, la RS despierta muchos más intereses, debido a la interacción con personas que se salen del contexto común, pero que a la vez están aprendiendo lo mismo y con los mismo objetivos.

8. ¿La didáctica y metodología fue atractiva y motivadora para realizar el trabajo por medio de la HT?

Metodológicamente se realizó una descripción de las HT de manera acorde con el nivel de los estudiantes, además la interacción permanente con los medios tecnológicos, motivo a explorar las mismas, aunque la RS por brindar interacción con pares de otras IE despertó mayor motivación que la $\mathrm{BD}$ donde comparten solo con estudiantes de la misma IE.

9. ¿La HT fue estable, diversa y útil para mejorar la calidad de la educación media técnica?

Las dos HT fueron aceptadas, adaptadas y aplicadas en los contextos; así mismo la utilización de una RS que articule las experiencias significativas, dio diversidad al proceso de enseñanza aprendizaje, lo que no sucedió con la BD que tuvo dificultad en estabilidad y no creó el criterio de utilidad en los estudiantes. Cabe resaltar que al responder la pregunta en cuanto a estabilidad, la RS sale mal librada, ya que la necesidad de conectividad permanente y de calidad no se encuentra en ninguna de las IE participes del proceso. 
Efrén Salgado Vivas, Marcela Georgina Gómez-Zermeño y María Manuela Pintor Chávez

10. ¿Existió manejo permanente de la HT acorde con el objetivo de mejorar la calidad de la educación media técnica?

Efectivamente, los docentes participantes de la investigación, estuvieron pendientes de los avances diarios de los estudiantes. Un concepto a tener en cuenta es la fácil distracción de los estudiantes al tener conectividad, interactuando por otros medios como Facebook y otras páginas web, desaprovechando el tiempo a utilizar en el aprendizaje.

\section{Encuesta de Satisfacción}

El tercer instrumento fue la encuesta referente al nivel de satisfacción que se tuvo del manejo de la biblioteca digital y redes sociales, lo que arrojo los siguientes resultados:

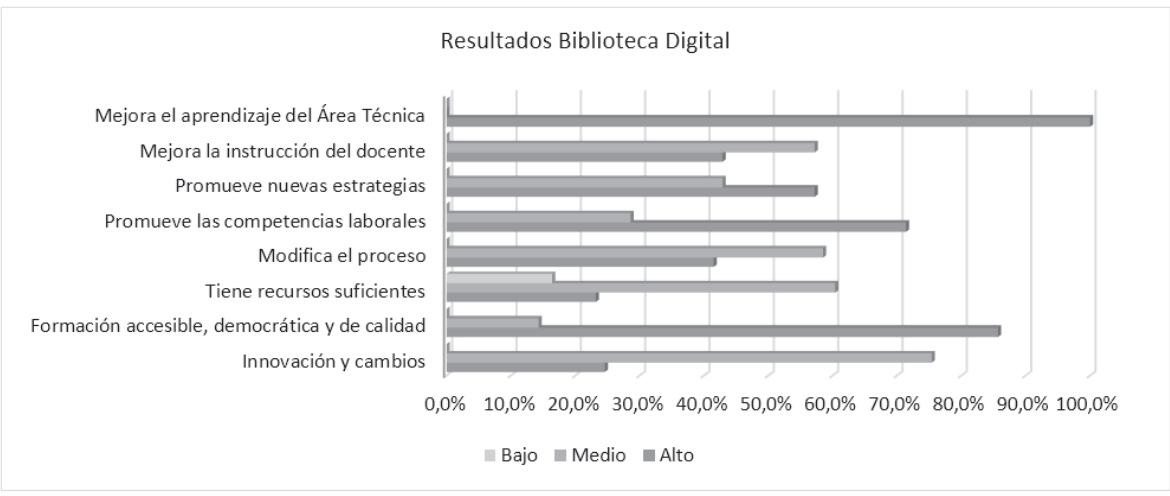

\section{Figura 4. Contexto Biblioteca Digital.}



Figura 5. Contexto Red Social. 
Educación rural y la adquisición de competencias laborales: una innovación mediante el uso de recursos digitales

Efrén Salgado Vivas, Marcela Georgina Gómez-Zermeño y María Manuela Pintor Chávez

Se indica que cualquier HT clara en objetivos, que modifique la forma tradicional de realizar el proceso de enseñanza - aprendizaje es buena para su implementación. Comparando las HT desde "el contexto", la $\mathrm{BD}$, en cuanto a implementación genera facilidades, debido a que no necesita conexión a internet y se limita a ser independiente en cada necesidad, frente a la RS que es más ambiciosa en compartir la información seleccionada en cada IE y que a su vez tiene mayor influencia para modificar el proceso.

Posteriormente, se analizaron diversos aspectos tanto de la biblioteca digital como redes sociales, con el objetivo de comparar la experiencia de los estudiantes. En cuanto a los objetivo de cada HT, la BD y RS fueron vistas como favorables para la integración de TIC y relevante para satisfacer las necesidades educativas de los estudiantes.

Se apreció que la BD es más adecuada para cubrir las necesidades educativas y la manera en que puede integrarse en las actividades académicas, y especialmente porque puede ser utilizada sin conexión a internet, contrario a las RS. Por otro lado, en cuanto a la diversidad de servicios, la BD no ofrece la diversidad que puede tener una RS integrada por mayor población, en donde los estudiantes a pesar de ser técnicos en áreas referentes a agropecuaria, existe la posibilidad de especializaciones como es el caso de la agroindustria, la producción pecuaria y otros. Lo que se analiza es una amplia ventaja de la RS en cuanto a diversidad frente a la BD.

Respecto a los contenidos, se nota que la RS ofrece más herramientas que la BD, ya que la RS permite utilizas foros, chat y otros, además los contenidos incluidos en la BD también se pueden incluir en la RS.

Al hacer comparación de las HT como estrategias de aprendizaje, tiene mayor aceptación la RS, ya que se puede interactuar, trabajar en grupo, fomentar el debate, brinda ejemplos entre estudiantes, se estimula la búsqueda de información y un sinfín de ventajas, frente a la BD digital que solo se limita a la acumulación de documentos, fotos, videos, etc., que también son de suma importancia para la mejora de la calidad de la educación.

Respecto a la usabilidad, Los usuarios expresaron que la biblioteca digital es apropiada para el manejo de imágenes, audio y video, así como el uso de lenguaje formal. En los demás puntos, la RS ofrece manejos diversos y amplios; demostraciones y alternativas para compartir publicaciones. La RS se presta para la discriminación por parte de los 
usuarios, debido al tipo de contenidos que pueden ser publicados. En cuanto a la accesibilidad, el acceso a la información y contenido se vieron favorecidos en ambos recursos, sin embargo el acceso a la práctica en la RS tuvo una baja respuesta, en comparación con la BD. Se analiza que el acceso a la BD no necesita conexión permanente a internet, en comparación con la RS que debe tener conexión estable y de calidad, hace que en este ítem la BD sea superior cuantitativamente.

\section{Nivel de satisfacción referente a la calidad de la herramienta tecnológica}

Cada estudiante califico de 1 a 10 el nivel de satisfacción del manejo de la HT donde 1 es muy bajo y 10 es lo más alto. Los 63 de la muestra, tienen conceptos relacionados frente a la satisfacción del uso y manejo de las HT; mientras que la mediana de la BD se encuentra en 4 y la moda es 6; la RS se encuentra en 8 y moda 9. Al profundizar algunos rangos bajos en la RS, la falta de conectividad generó inconformidad al no entender que el problema es de la empresa que presta el servicio de internet y no de la HT. Es importante tener en cuenta que a pesar de estar en zonas alejadas del urbanismo y la modernidad, los estudiantes, padres y comunidad educativa en general, se preocupan por mejorar el conocimiento de TIC. Los docentes en este momento tienen un dilema frente a su preparación en el manejo de la tecnología, pues si bien han recibido algunas capacitaciones por entidades, como menciona, están utilizando las tecnologías del mañana para impartir el currículum del pasado (Correa y De Pablos, 2009).

\section{Conclusiones}

Una vez terminada la investigación y analizada toda la información en cuanto a diseño, contenido, pedagogías y tecnologías, se presenta algunas conclusiones y reflexiones para el mejoramiento de la calidad de la educación en el sector rural con la utilización de HT.

El instrumento cuestionario de caracterización permitió conocer el perfil poblacional, donde se percibió que la calidad en las IE participantes es baja; las personas adultas tienen clara la importancia del co- 
Educación rural y la adquisición de competencias laborales: una innovación mediante el uso de recursos digitales

Efrén Salgado Vivas, Marcela Georgina Gómez-Zermeño y María Manuela Pintor Chávez

nocimiento globalizado y de interactuar con otras culturas, pero tienen miedo a los contenidos no favorables para los estudiantes; la capacitación a estudiantes o docentes sobre el manejo del internet y de recursos educativos es baja o nula, existen deficiencias de seguimiento y control en la implementación de equipos tecnológicos y no existen guías institucionales sobre el manejo de TIC en Educación. Por otra parte, las entrevistas a los participantes en grupo focal permitió identificar las expectativas del uso de las herramientas tecnológicas de los estudiantes, en cuanto a la calidad de la Herramienta Tecnológica, la calidad didáctica y metodológica y la Calidad Técnica, destacando la aceptación, adaptación y aplicación de las dos HT, en los contextos del estudio. Finalmente, la encuesta de satisfacción nos permitió identificar que a pesar de estar en zonas relativamente alejadas del urbanismo y la modernidad, los estudiantes, padres y comunidad educativa en general, se preocupan por mejorar el conocimiento de nuevas las TIC.

El deseo de aprender en un contexto globalizado por parte de los estudiantes es cada vez más elevada, se debe tener en cuenta que a pesar de estar en contextos rurales, al haber conectividad se puede interactuar con el mundo entero y eso lo deben tener en cuenta las entidades gubernamentales para permitir que los estudiantes lleven el proceso de aprendizaje de forma dinámica y concreta hacia los interés particulares.

Los docentes en este momento tienen un dilema frente a su preparación en el manejo de los recursos tecnológicos, pues si bien han recibido algunas capacitaciones por entidades como Computadores para Educar en el manejo de las TIC; como se cita en el capítulo 2 "se están utilizando la tecnología del mañana para impartir el curriculum del pasado (Correa \& De Pablos, 2009)".

Según Busquets (2013), el nuevo rol del docente frente a TIC lo obliga a modificar la educación a un aprendizaje con creatividad inspirado en las necesidades del contexto, crear experiencias de aprendizaje que motiven a la implementación de lo aprendido en la real y promover el manejo de HT con responsabilidad. Los resultados, llevan a la construcción de tres criterios que influyen en la implementación y manejo de las redes sociales como herramientas tecnológicas, que corresponden a una realidad contextualizada en el sector rural del Departamento de Cundinamarca. 


\section{-Primer Criterio}

Dentro del contexto de la educación rural, el desarrollo de competencias generales y específicas permiten que los jóvenes se desempeñen de manera eficiente como seres involucrados en procesos productivos (MEN, 2007). De este modo, el tema que se abordó es relevante para el mejoramiento de las condiciones básicas de la población rural, ya que la ampliación y actualización del conocimiento en nuevas tecnológicas y métodos de producción son necesarios no solamente en el Departamento de Cundinamarca, sino en todos los sectores dedicados a la producción, transformación y comercialización de bienes y servicios provenientes de la agricultura y la explotación animal.

Asimismo, las herramientas tecnológicas brindan la opción a los estudiantes de auto gestionar su propio conocimiento y la puesta en práctica de habilidades, facilitando la posibilidad de trascender en el contexto inmediato para actuar y adaptarse a las verdaderas necesidades de progreso y desarrollo tanto personal como comunitario. Por ello las redes sociales, como herramienta tecnológica y pedagógica, debe ofrecer la posibilidad de interacción entre pares de las IE que manejan contenidos similares en formación de competencias laborales, generando redes de aprendizaje.

\section{-Segundo Criterio}

Los contenidos para la interacción de los estudiantes de diferentes IE debe manejar temas relevantes, se debe contar con un diseñador instruccional que genere debate e interés en las discusiones en foros, chat y demás interacciones tanto sincrónicas como asincrónicas, esto para evitar ambigüedades, duplicidad de contenidos o mala utilización del recurso.

\section{-Tercer Criterio}

La estructura tecnológica, ya que existen fallas en la conectividad que limitan el manejo de los recursos tecnológicos en el contexto rural. Se debe gestionar una conectividad de calidad, al igual que la calidad de los medios tecnológicos (computadoras y tablet). Se debe reafirmar el compromiso de entidades por ofrecer conectividad continua y de calidad, recordando que cuando una IE cuenta con buen número de computado- 
Educación rural y la adquisición de competencias laborales: una innovación mediante el uso de recursos digitales

Efrén Salgado Vivas, Marcela Georgina Gómez-Zermeño y María Manuela Pintor Chávez

ras a disposición y buen acceso a la web, se permite generar procesos constructivos, enriquecedores e interesantes (Valencia y Camargo, 2013).

Adicionalmente, se determinó que la Biblioteca Digital puede ser una herramienta tecnológica complementaria a la Red Social Educativa, es por eso que se propone la gestión para la construcción dentro de la "Red Social Educativa de Cundinamarca" una sección única y exclusiva para las Áreas Técnicas, en especial las afines a la agropecuaria; donde coincidan docentes, estudiantes, padres de familia y demás actores involucrados el proceso de enseñanza aprendizaje y sea utilizada como herramienta tecnológica y de innovación; para fortalecer las prácticas en la educación media técnica y el aprendizaje de las competencias laborales en las áreas de producción en las IE rurales del Departamento de Cundinamarca.

Se recomienda a Instituciones y docentes, planificar la formación permanente, pertinente y actualizada que fortalezca el uso de TIC que facilite la práctica educativa desde el aprendizaje significativo y lograr impacto positivo en la calidad de la educación. De igual forma, se exhorta a propiciar interacciones tecnológicas entre los actores educativos, donde el docente sea generador de cambio, rompiendo con esquemas tradicionales.

Es importante crear un código de ética para el manejo de recursos tecnológicos en las instituciones educativas, ya que los estudiantes ven la utilización del internet más como medio de esparcimiento, ocio y entretenimiento, en vez de considerarlo para la formación lúdica-pedagógica y la construcción del conocimiento. Respecto a este punto, es importante valorar la Web 2.0 con herramienta tecnológica que permite el intercambio académico eficaz en entornos de aprendizaje y mejoramiento de las competencias laborales.

\section{Referencias bibliográficas}

Alemán, L. Y., Gómez-Zermeño, M. G., Parada, E. \& Sáenz, P. (2011). Extracurricular strategies for teaching innovation. En A. Mateos, M. Anderson \& J. M.Rodríguezz (Eds.) KickStart. New ways of teaching innovation (pp. 327-369). Salamanca, España: Amarú Editores.

Barber, M. y Mourshed, M. (2008). Como hicieron los sistemas educativos con mejor desempeño del mundo para alcanzar sus objetivos. New York: McKinsey Compañy.

Busquets, F. (2013). Nuevo rol docente frente a las TICs. Recuperado de http://www.educarchile.cl/ech/pro/app/detalle?id=76656 
Educación rural y la adquisición de competencias laborales: una innovación mediante el uso de recursos digitales

Efrén Salgado Vivas, Marcela Georgina Gómez-Zermeño y María Manuela Pintor Chávez

Cabero, J. (2006). Bases Pedagogicas del e-learning. Revista de Universidad y Sociedad del Conocimiento, 3(1). Recuperado de http://www.uoc.edu/rusc/3/1/dt/esp/cabero. pdf

Correa, J. M., y De Pablos, J. (2009). Nuevas Tecnologías e Innovación Educativa. Revista de Psicodidáctica, 14(1), 133 - 145. Recuperado de http://www.redalyc.org/articulo. oa?id=17512723009

Cuesta, O. J. (2008). Reflexiones sobre la Educación Rural en el Marco de la Comunicacion - Educación. Civilizar, 8(15), 89-102. Recuperadode http://redalyc.uaemex.mx/ src/inicio/ArtPdfRed.jsp?iCve=100212243007

Gómez-Zermeño, M. G. (2009). Estudio exploratorio-descriptivo de competencias interculturales en instructores comunitarios del Consejo Nacional de Fomento Educativo que brinda servicio en la modalidad de atención educativa a población educativa en el Estado de Chiapas. Monterrey, Nuevo Leon, Mexico: Tecnologico de Monterrey.

Leibovich, J., Nigrinis, M., y Ramos, M. (2005). Caracterización del Mercado Laboral en Colombia. Bogota - Colombia: Banco de la Republica.

McKinsey \& Company (2007). Cómo hicieron los sistemas educativos con mejor desempeño del mundo para alcanzar sus objetivo. Recuperado de https://mckinseyonsociety.com/downloads/reports/Education/Como_hicieron_los_sistemas_educativos.pdf

Ministerio de Educacion Nacional [MEN] (2002). Escuelas Normales Superiores - Mas Cerca del Sector Rural. Al Tablero(15), págs. 2-5. Recuperado de http://www.mineducacion.gov.co/1621/article-87236.html

Ministerio de Educacion Nacional [MEN]. (2007). Competencias Laborales Generales. Serie Guias, 21.

Ospina, J. E. (2008). Encicloperia Agropecuaria. Bogota: Terranova.

Portal Colombia Aprende. (2007). Colombia Aprende. Recuperado de http://www.colombiaaprende.edu.co/html/mediateca/1607/articles-175836_archivo.pdf

Rimari, W. (1996). La Innovación Educativa - Un Instrumento de Desarrollo. Revista Pedagogica.

Santiesteban, D. F. (2008). Colombia Frente al Posible Tratado de Libre Comercio con Estados Unidos. Bucaramanga - Colombia: Porter.

Schmelkes, S. (1994). Hacia una Mejor Calidad de Nuestras Escuelas. Mexico: Interamer 32 Serie Educativa.

Stake, R. (2007). Investigacion con Estudios de Caso. Madrid, España: Morata.

Tramullas, J (2011). Bibliotecas Digitales. Madrid, España: Depto de Ciencias de la Documentación. Universidad de Zaragoza.

Valencia, J. y Camargo, F. (2013). Estrategias para el Fortalecimiento de las TIC en las Instituciones Educativas Oficiales de Colombia. Colombia Digital. Recuperado de http:// colombiadigital.net/publicaciones_ccd/descargar_publicaciones.php?publi=18

Valenzuela, J. R., y Flores, M. (2011). Fundamentos de Investigación Educativa (Vol. 2). Monterey, Mexico: Editorial Digital del Tecnologico de Monterrey.

Vargas, L., Gómez-Zermeño, M., y Gómez-Zermeño, R. (2013). Desarrollo de Habilidades Cognitivas y Tecnologicas con Aprendizaje Movil. Revista de Investigación Educativa de la Escuela de Graduados en Educación, 3(6), 30-39. Recuperado de http:// rieege.tecvirtual.mx/index.php/rieege/article/view/76 
